\title{
COMPUTATIONAL SIMULATION OF VISUAL DISTRACTION EFFECTS ON CAR DRIVERS' SituATION AWARENESS
}

\author{
Thierry Bellet ${ }^{1}$, Jean-Charles Bornard ${ }^{1,3}$, Pierre Mayenobe ${ }^{1}$, Jean-Christophe Paris ${ }^{1,3}$, \\ Dominique Gruyer ${ }^{2}$, Bernard Claverie ${ }^{3}$ \\ ${ }^{1}$ IFSTTAR (LESCOT), 25 Avenue François Mitterrand, 69675 Bron cedex, France \\ ${ }^{2}$ IFSTTAR (LIVIC), 14 Route de la minière, 78000 Versailles, France \\ ${ }^{3}$ ENSC (CIH), 146, rue Léo Saignat, 33076 Bordeaux, France \\ (corresponding author : thierry.bellet@ifsttar.fr)
}

\begin{abstract}
This paper presents a computational modeling approach for negative effects simulation of visual distraction while driving a car. In order to investigate these effects, an experiment was firstly implemented on a driving simulator. Twenty participants were invited to perform a car following task in different driving conditions (12 driving scenarios), with or without a secondary task of visual distraction. Empirical data collected through this experiment show that visual distraction negatively impacts the driving performance at both perceptive and behavioral levels, and then increase the risk having a crash. Beyond these effects on the observable performance, the aim of this study is also to investigate and simulate such distractive effects on mental models of the road environment. Indeed, driver's decisions and behaviors are based on a temporal-spatial mental model, corresponding to the driver's situational awareness (SA). This mental representation must be permanently updated by perceptive information extracted in the road scene to be efficient. In case of visual distraction requiring off-road scanning, mental model updating is un-perfectly done and driver's actions are thus based on a mental representation that can dramatically differ of the situational reality, in case of a critical change in the traffic conditions (e.g. sudden braking in the lead car). From these empirical results, a computational model (named COSMODRIVE for COgnitive Simulation MOdel of the DRIVEr) was implemented for simulating visual distraction effects and human errors risks at perceptive (visual scanning changes) cognitive (erroneous Situational Awareness) and behavioral levels (late reaction time and crash risk increasing).
\end{abstract}

Keywords: Computational Model, car Driver, Visual Distraction, Situation Awareness, Temporal-Spatial Mental Representation.

\section{Introduction: Visual distraction and research objective in terms of computational simulation}

Driving requires visual attention in order to safely control the car and to respond to events happening on the road. Driver distraction occurs when a driver is delayed in the recognition of information needed to safely accomplish the driving task because some event, activity, object, or person within or outside the vehicle compelled or tended to induce the driver's shifting attention away from the driving task (Treat, 1980). In the same way, distraction has been more recently defined by Lee, Regan and Young (2009) as a diversion of attention away from activities critical for safe driving toward a competing activity. Two main forms of distraction are commonly described in the literature, namely visual and cognitive distraction. The former takes a driver's "eyes-off-road", while the latter takes their "minds-offroad" (Victor et al., 2005). The present study is only focus on visual distraction due to a secondary visual task taking the driver's eyes off the road. Such type of visual distraction can occurs when driver look at in-vehicle display system.
For example, in research conducted by Wierwille et al. (1988) under real traffic conditions where text was displayed on an on-board screen, the average length of a glance at the outside environment was 1.5 to 1.7 seconds, while the amount of time spent watching the road decreased to about 50 to $65 \%$ of total eye movement. Visual scanning toward on-board-devices varies with the nature of displayed information and the type of additional task to be performed while driving, but also according to the situational demand and driver's adaptation strategies regarding both the driving situation and the demand and the reading task demands. However, focusing visual attention for some period of time on in-vehicle visual target can creates an unsafe driving issue. Senders et al. (1967) argued that when drivers look away from the road, uncertainty about the roadway situation increases. When uncertainty reaches a certain threshold, drivers look back to the road. More recently, Wierwille (1993) quantified this threshold of off-road glance duration at 1.8 seconds on a straight road and 1.2 seconds on a curve on average for a normal driver. Such thresholds may also vary according to driver speed or traffic condition and may be also subject to individual differences. Changes in driver behavior due to visual distraction have been identified in simulator or in vivo studies. Several studies have shown that visual distraction increases the dispersion of eye gaze pattern from the roadway (e.g. Donmez et al., 2007). In terms of driving performance, visual distraction has been also associated in the literature with large, discrete steering adjustments and increased lane deviations (e.g. Engström, Johansson and Östlund, 2005). However, as discussed by Zhang (2011), such inference has been mainly assessed for lower-level of driving control and less is known concerning the internal cognitive effect of visual distraction. Moreover, as explained this author, a data-driven approach to identifying detrimental effects of distraction may not be sufficient to establish a causal link between driver performance and visual process interference. Modeling internal changes in driver Situation Awareness of the driving environment due to visual distraction are required to conclusively identify precise relationships between drivers' performances and distraction and support effective mitigation strategies for distractions. This is typically what this research would like to explore. Beyond the well-known impact of visual distractions on drivers visual strategies and driving performance at the operational level, the aim of this research is above all to develop a computational model able to simulate these distractive effects at the internal cognitive level corresponding to drivers' Situational Awareness. 


\section{Empirical data collection among human drivers to study visual distraction effects}

The methodological specificity of the driver modelling approach implemented in this research was to use the same virtual Platform (named SIVIC; Gruyer et al., 2006) as (i) a driving simulator for empirical data collection among human drivers, and then, as (ii) a virtual road environment to be interfaced with the driver model for virtual simulations (in charge to reproduce humans' performances). According to this approach, human drivers' behaviour and driver model performances were observed and simulated for the same driving scenarios, in the same virtual road environment.

\subsection{Apparatus}

The experiment used is a fixed-base simulator integrating a real car seat, three PC monitors for presenting the driving scene (the back mirror view is computationally integrated in the central image), and a Logitec G 25 kit including the steering wheels, the 3 pedals, a gear box, and the indicators commands. Two web-cameras are used for recording the driver's face and the feet movement on the pedals. A third video camera was also added behind the car seat, in order to film the driving environment and the driver's cockpit activity. A 12-inch tablet computer was placed in front of the main simulator screens. This tablet was used to present the visual distraction tasks to drivers. The screen was positioned approximately 15 degrees down and 30 degree right of the natural line of sight of participants in viewing the driving scene.

\subsection{Participants}

Twenty experienced drivers of middle-age (from 23 to 56 years old) participated to this experiment. All the drivers have a minimum of 5 years of driving experience and they drive a minimum of $5.000 \mathrm{~km}$ per year. The recruitment of subjects was balanced for gender. Participants were instructed to perform the secondary task in accordance with the demands of the driving situation. The instruction emphasized that safe driving was of the highest priority.

\subsection{Driving task}

The full experiment followed a $1 \times 3 \times 2 \times 2 \times 2$ factorial design with one primary driving task of car following to be performed in three different driving contexts (requiring different driving speeds: $130 \mathrm{~km} / \mathrm{h}$ for Highway, $90 \mathrm{~km} / \mathrm{h}$ for rural roads and $50 \mathrm{~km} / \mathrm{h}$ for urban areas), from two required following distances (free versus imposed at a value of 0.6 second of Inter-Vehicular Time [IVT]), and two types of lead car behavior (having a steady versus irregular velocity) and then, two levels of visual distraction (with and without). In total, there were 12 driving scenarios to which each participant was exposed, once time without any secondary task, and then, on time with a secondary task. Each scenario was around 1 minute in duration and presented one experimental condition.

\subsection{Visual secondary task}

The Secondary Task of visual distraction to be performed by the participants was the following: a set of 3 visual pictograms, associated with an auditory beep, were displayed on an additional screen situated on the right side (near the usual position of the radio). Some seconds later (from 3 to $4 \mathrm{sec}$.), 1 of this 3 pictograms appeared under the first set, and the driver had to use a 3-buttons command for indicating which pictogram is replicated (Fig. 1).

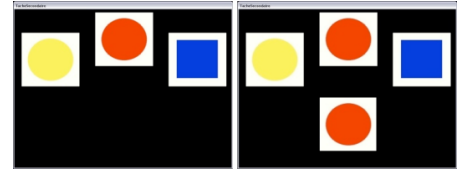

Figure 1: The visual Secondary Task to be performed

\subsection{Main results}

\subsubsection{Visual strategies for additional screen scanning}

Visual strategies during secondary task have been extracted from the analysis of video film of participants' faces collected during the experiment. The two main different visual scanning patterns of the additional-screen observed among human drivers are presented in figure 2 (others strategies are adaptations of one of the two main patterns).

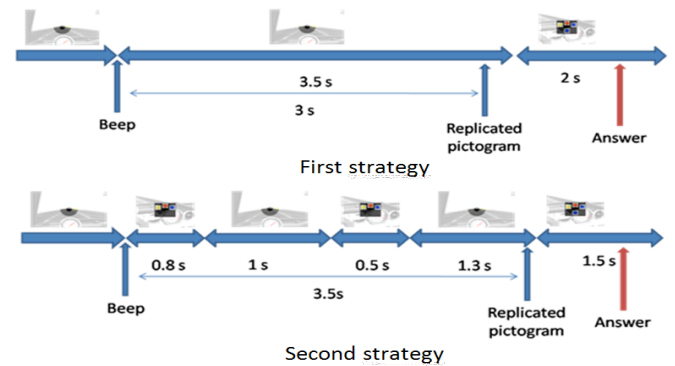

Figure 2: Visual scanning patterns observed among human drivers

The first strategy (58\% of the cases) consists in waiting from 3 to 4 seconds when the beep occurs, hoping that only one scanning of the screen will allow the participant (a) to see the 3 pictograms, (b) to see the replicated one, and (c) to provide the answer. With this strategy, only a long glance of 2 seconds (in mean) is generally needed. The second strategy (observed in $31 \%$ of the cases) was to look at the screen briefly (mean glance duration of $0.8 \mathrm{sec}$.) when the beep occurs in order to observe the 3 pictograms, and then to go back to the road scene while regularly checking the screen (via brief glances of $0.5 \mathrm{sec}$ ) until the replicated pictogram appears. When it occurs, a more long off-road glance (around $1.5 \mathrm{sec}$.) is implemented for both checking the replicated pictogram and validating the answer.

\subsubsection{Visual distraction effect on driving performance}

Two main negative impacts of a visual distraction on the drivers' performances were observed during this experiment. The first one occurs in normal conditions, and the second one occurs for critical scenarios (i.e. when the lead car brakes), increasing the accident risk. 
In normal driving conditions, two main differences due to visual distraction were observed among the participants: (i) a significant reduction (T-test, $\mathrm{p}<0.001$ ) of the safety margins in free following conditions (without ST, mean value of IVT is of $3 \mathrm{~s}$. without ST, vs $2.65 \mathrm{~s}$. with ST) and (ii) a significant degradation $(\mathrm{p}<0.05)$ of the following performance in constrained following conditions (in these scenarios, drivers have to follow the lead car at an imposed IVT of $0.6 \mathrm{~s}$., and the percentage of time when this value is performed is of $57 \%$ without ST, vs $44 \%$ with ST). These results show a negative effect of visual distraction for short following distance keeping.

In critical driving conditions, the two main negative impacts of the visual ST on drivers' performances are (i) an increasing of reaction time for braking (the differences are only significant for the constrained following task : $0.89 \mathrm{~s}$. vs $1.1 \mathrm{~s}$.; $\mathrm{p}<0.05$ ), and (ii) a risk of crash increasing. The Table 1 presents the percentages of collision occurring with the lead car for the total number of required emergency braking, by respectively considering the different driving scenarios investigated. It appears that the risk of collision due to a visual distraction is here significantly increased for 4 of the 10 driving scenarios requiring an emergency braking (i.e. bold values). The highest negative impacts of visual ST were observed for the constrained unsteady car following scenarios, in both urban and rural environments.

Table 1: Percentages of collision with the lead car

\begin{tabular}{|c|c|c|c|}
\hline Context & Driving scenario & No ST & With ST \\
\hline Highway & Free steady lead car following & $55 \%$ & $50 \%$ \\
\hline & Free unsteady lead car following* & $\mathbf{3 5 \%}$ & $\mathbf{5 0} \%$ \\
\hline & Constrained steady lead car following & $65 \%$ & $70 \%$ \\
\hline & Constrained unsteady lead car following & $70 \%$ & $70 \%$ \\
\hline Rural & Free unsteady lead car following & $60 \%$ & $60 \%$ \\
\hline & Constrained unsteady lead car following* & $\mathbf{5 5 \%}$ & $\mathbf{8 0} \%$ \\
\hline Urban & Free steady car lead following* & $\mathbf{2 0} \%$ & $\mathbf{3 0} \%$ \\
\hline & Free unsteady lead car following & $30 \%$ & $30 \%$ \\
\hline & Constrained steady lead car following & $30 \%$ & $30 \%$ \\
\hline & Constrained unsteady lead car following* & $\mathbf{2 5 \%}$ & $\mathbf{9 0} \%$ \\
\hline
\end{tabular}

$\left({ }^{*}\right.$ Bold Yellow Values indicate statistical significant differences in driving performance due to visual distraction; T-test, $p<0.05$ )

\subsubsection{Example of crash due to visual distraction}

The following figure presents a typical case of driving accident due to visual distraction, as observed during this experiment (in free following conditions, view $a$ ). In this example, the lead car brakes when the driver is looking for the additional screen (view $b$ ), via a long glance of 2 seconds. When she repays attention to the road (view $c$ ), she however discovers a critical gap between the expected position of the lead car as mentally assessed during the offroad glance (by assuming a steady speed of the lead car during this period) and the objective reality where the lead car is actually very close. Therefore, she immediately carried out an emergency braking ( 0.78 second of reaction time). Unfortunately, the collision cannot be avoided, and the crash with the lead car occurs in view $d$.

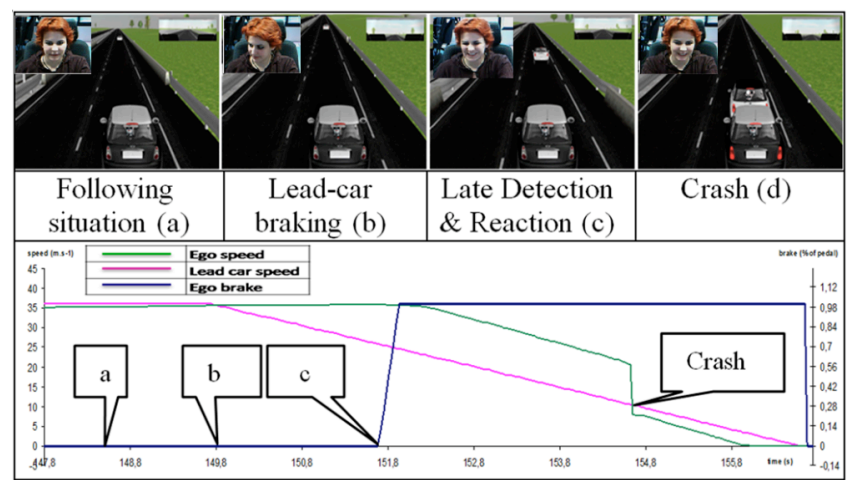

Figure 3: Typical example of crash due to visual distraction

\section{Computational modeling and simulation of visual distraction effects on drivers' $S A$}

By using the empirical data collected in this experiment, a computational model, based on the COSMODRIVE (COgnitive Simulation MOdel of the DRIVEr) theoretical approach (Bellet et al., 2007), has been implemented into the SIVIC virtual plate-form (Gruyer et al, 2006). By contrast with other driver models available in the literature, the core specificity of COSMODRIVE is to simulate drivers' mental representation as a temporal-spatial (i.e. 3 Dimensional) and dynamic model of the road environment. Indeed, from their interaction with the road environment, drivers build mental model of events and objects surrounding them. This mental model corresponds to the driver's Situation Awareness (Endsley, 1995). They are dynamically formulated in working memory through a matching process between perceived information and preexisting operative knowledge (Ochanine, 1977). At the tactical level (Michon, 1985), such a mental representations provides an ego-centred and a goal-oriented understanding of the traffic situation. They take the form of a dynamic 3D model of the road environment, liable to be mentally explored by the driver in order to anticipate events or action effects through cognitive simulations of mental deployment (Bellet et al, 2010), and thus providing expectations on future situational states. This cognitive process of anticipation, based on both implicit and explicit mental simulations (Bellet et al., 2009), is a core function of the human cognitive system in dynamic contexts. The central structure supporting to the driver's SA in COSMODRIVE cognitive architecture is working memory. From this point of view, this architecture is inspired by the ACT-R theory (Anderson et al., 2006). However, the working memory of COSMODRIVE merges both procedural and declarative memories, and comes more from the operational memory concept of Zintchenko (1966) than from the Baddeley's working memory model (1986). With COMSODRIVE, car driving is modeling as a dynamic regulation loop of interaction between drivers and the road environment. 
Figure 4 provides a synthetic overview of this model as implemented on the SIVIC virtual platform. Synthetically the functional architecture of the model is based on 3 main modules (i.e. Perception, Cognition, and Action modules), in order to drive a virtual car into a virtual environment, through two synchronized "Perception-Cognition-Action" regulation loops: an automatic and implicit mode versus an attentional and explicit mode (Bellet et al, 2009). This dichotomy is well established in scientific literature, for example, with the distinction put forward by Schneider and Schiffrin (1977) between controlled processes, which require cognitive resources and which can only be performed sequentially, and automatic processes, which can be performed in parallel without any attentional effort. In the same way, Rasmussen (1986) distinguishes different levels of activity control according to whether the behaviours implemented rely on (i) highly integrated sensorial-motor reflexes (Skill-based behaviors), (ii) well mastered decision rules for managing familiar situations (Rule-based behaviors), or (iii) more generic knowledge that is activated in new situations, for which the driver have not any prior experience (Knowledge-based behaviors).

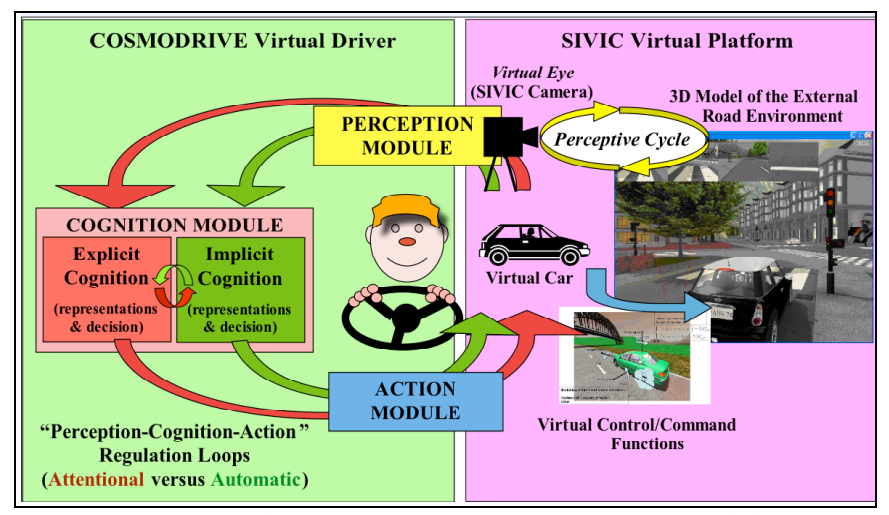

Fig. 4: Architecture of COSMODRIVE model

From this architecture, the Perception Module is in charge to simulate human information processing, the Cognition Module is in charge to simulate mental representation elaboration (SA) and decision-making processes at both the attentional and automatic levels, and the Action Module is in charge to simulate executive functions and vehicle control abilities, allowing the model to dynamically progress on the SIVIC virtual road by driving a virtual car.

\subsection{The Perception module}

The Perception Module acts as an "interface" between the external road environment (as simulated with SiVIC) and the driver model. It simulates human information processing of sensorial data before their integration in the Cognition module for traffic conditions analysis, situational change anticipation, decision making, and then action planning and implementation through the Action Module. The Perception module is based on a virtual eye (Figure 5). This virtual eye includes three visual field zones: the central zone corresponding to foveal vision (solid angle of $2.5^{\circ}$ centred on the fixation point) with a high visual acuity, para-foveal vision (from $2.5^{\circ}$ to $9^{\circ}$ ), and peripheral vision (from $9^{\circ}$ to $150^{\circ}$ ), allowing only the perception of dynamic events.

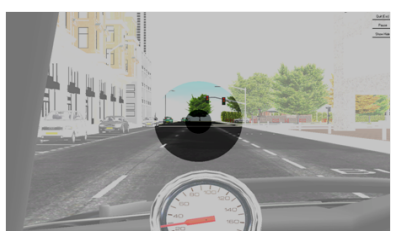

Fig. 5: COSMODRIVE virtual eye

From this virtual eye, COSMODRIVE is able to integrate information extracted in the road environment through two main processes (Bornard et al, 2011). The first one, named perceptive integration, is a data-driven process (i.e. bottomup) and allows the cognitive integration of environmental information in the driver's mental representations. The second one, named perceptive exploration and based on Neisser's perceptual cycle theory (1976), is a "knowledgedriven" process (i.e. top-down) in charge to actively explore the road scene, according to the tactical goal to be reached and to the event expectations included in the driving schemas. In the frame of a car following task, the main point of interest of the driver's visual attention is the lead car. However, in case of a visual secondary task to be performed while driving, the virtual eye must sometimes leave the road in order to observe the additional screen, according to the 2 different visual scanning patterns observed among human drivers during our experiment (presented in fig. 2).

\subsection{The Cognition module}

The Cognition Module in charge to simulate human drivers' abilities in Situational Awareness (i.e. mental representation elaboration), Decision-making and Action planning. Mental Representation elaboration in COSMODRIVE is firstly based on a tactical driving schemas. Coming from both the Piaget's (1936) concept of operative scheme and the Minsky (1975) frames theory, driving schema is a computational formalism defined at IFSTTAR for driving knowledge modeling at the tactical level (Bellet et al, 1999; 2007). It is a functional model of the road Infrastructure associated with a Tactical Goal to be reached in this context. It is made of a Driving Path, defined as a sequence of Driving Zones, and of a sequences of Actions to be implemented. Actions implementation depends of Conditions to be checked regarding the occurrence of Events in certain Perceptive Zones of the infrastructure. An event is an Object with specific Characteristics (its aspect, behaviour, or status). The term object is used here in its widest meaning. It can be a vehicle, a pedestrian, or a road sign. Once activated in working memory and instantiated with the characteristics of the current road environment, the active driving schema becomes the tactical mental representation of the driver, that is continuously updated as and when s/he progresses on the road. It corresponds to the driver's explicit awareness of the situation. In the frame of a car-following task on straight line, the driving schema is limited to the tactical goal of 
progressing along the same road lane (no overtaking), at a given speed, and keeping a safe distance with the lead car.

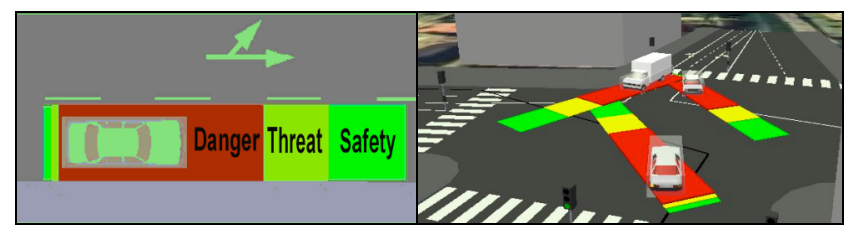

Figure 6: COSMODRIVE "Envelope-Zones" model

At the operational level, corresponding to an automatic control loop, COSMODRIVE regulation strategy is jointly based on envelope zones and pure pursuit point approaches. From a theoretical point of view, the concept of envelope zones comes from two classical theories in psychology: the notion of body image of Schilder (1950), and the theory of proxemics defined by Hall (1966), relating to the distance keeping in social interactions with other humans. Regarding car-driving activity, envelope zones also refer to safety margins. At this last level, COSMODRIVE model (Fig.6) is based on Kontaratos' work (1974) distinguishing a safety zone, a threat zone, and a danger zone. Envelope zones correspond to the portion of the path of driving schema to be occupied by the vehicle in the near future. As an "hidden dimension" of the social cognition, as suggested by Hall's theory (1966), these proxemics zones are also mentally projected to other road users, and are then used to dynamically interact with them, as well as to anticipate and manage the collision risks. This "virtual skin" is permanently active while driving, as an implicit awareness of our expected allocated space for moving. As with the Schilder's body schema, it belongs to a highly integrated cognitive level (i.e. implicit regulation loop), but at the same time, it favors the emergence of critical events in the driver's explicit awareness. Therefore, the envelope zones play a central role in the regulation of "social" as well as "physical" interactions with other road users under normal driving conditions (e .g. inter-vehicle distance keeping), and in the risk assessment of path conflicts and their management, if a critical situation occurs (commitment of emergency reactions).

Moreover, two Decision-Making processes are implemented in COSMODRIVE model, one for each regulation loops presented in fig. 4. At the attentional level, corresponding to explicit decisions, this process is modelling through StateTransition automats intimately linked with the driving path and conditions integrated in tactical driving schemas. In real driving conditions, this tactical level is typically used for overtaking decision-making. However, in the frame of the empirical data collected in our experiment, primarily involving automatic driving abilities, the tactical level is mainly active when the lead car suddenly brakes and when the situation becomes critical. At the automatic level, an implicit decision-making is implemented through envelope zones, in order to keep a safety distance with the lead car (i.e. keep it in the green zone).

\subsection{The Action module}

The Action Module is in charge to perform vehicle-control skills, according to the driving actions decided and planned at the representational level by the Cognition module. The two core regulation mechanisms effectively implemented by the Action Module are based on (i) the Pure-Pursuit Point method and (ii) safety margin keeping by using EnvelopeZones. The Pure Pursuit Point method is used by COSMODRIVE for the lateral and the longitudinal controls of the car along the driving path of a tactical schema (Mayenobe, 2004). Mathematically, the pure-pursuit point is defined as the intersection of the desired vehicle path and a circle of radius centered at the vehicle's rear axle midpoint (assuming front wheel steer). Intuitively, this point describes the steering curvature that would bring the vehicle to the desired lateral offset after traveling a distance of approximately 1 . Thus the position of the pure-pursuit point maps directly onto a recommended steering curvature: $\mathrm{k}=$ $2 \mathrm{x} / 1$, where $k$ is the curvature (reciprocal of steering radius), $x$ is the relative lateral offset to the pure-pursuit point in vehicle coordinates, and $l$ is a parameter known as the lookahead distance. According to this definition, the operational control of the car by COSMODRIVE is a monitoring loop in charge to permanently keep the Pursuit Point in the driving path, to a given speed assigned with each segment of the tactical schema, as instantiated in working memory.

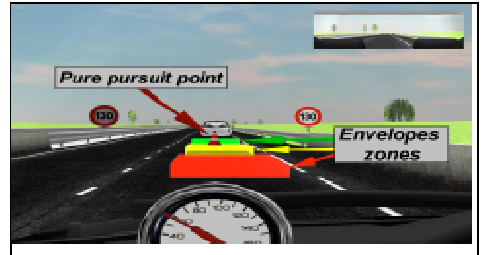

Figure 7: Pursuit Point and Envelope Zones

COSMODRIVE abilities for vehicle-control are thus supported in the Action module by the pure-pursuit point method (for monitoring the lateral and longitudinal position of the car), and by the envelope zones strategies (for managing interactions with the other road users). Figure 6 illustrates this regulation strategy in the frame of a carfollowing task: the pursuit point determines the cap to be followed by the virtual ego-car, and the envelope zones are used for keeping a safe IVT distance with the lead car.

\subsection{Simulation of visual distraction effects}

By considering the empirical data presented in section 2, the visual scanning patterns of the additional screen collected during this experiment among human drivers (cf. fig 2) were implemented in the Perception module of COSMODRIVE, in order to simulate visual distraction effects on drivers' behaviors (visual strategies and vehicle control) and to investigate human errors liable to occur when drivers perform a visual secondary task while driving. Indeed, beyond the observable effects of visual distraction on drivers' performance, the aim of the COSMODRIVE 
computational modeling approach was also to simulate such distractive impacts on car drivers Situational Awareness.

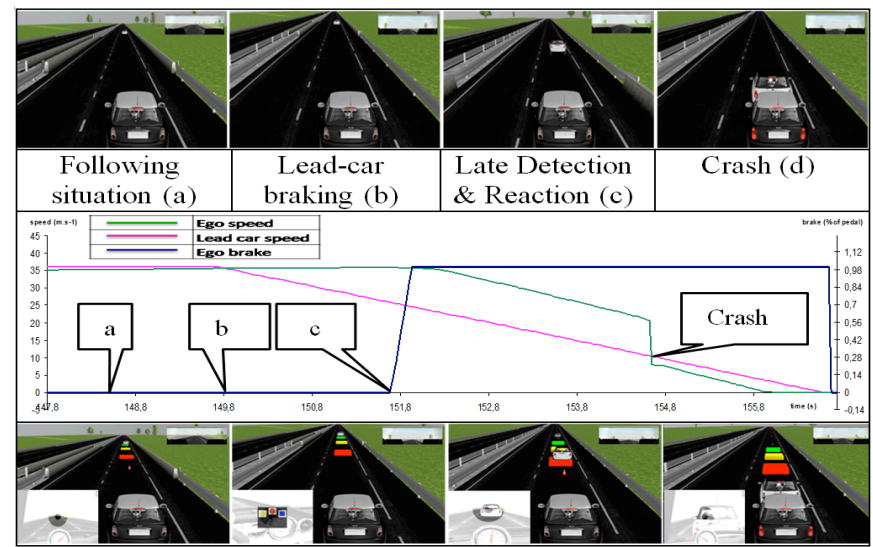

Fig. 8: driving performance simulation of a distracted driver

When driving, humans must continually update their mental model of the driving situation as and when they dynamically progress on the road. In case of additional task requiring offroad scanning, mental model updating is un-perfectly done and driver's actions are thus based on a mental representation that can dramatically differ of the situational reality, in case of a critical change in the traffic conditions.

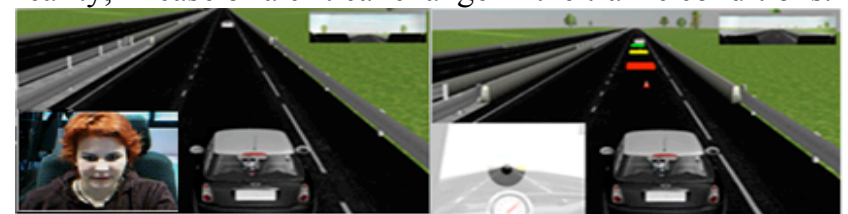

Stage 1: car following situation (left) and driver's SA (right)

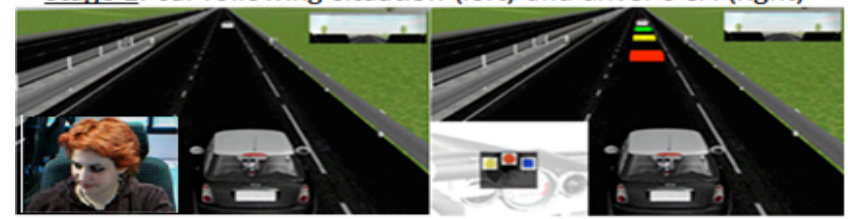

Stage 2: beginning of driver's on-board screen visual scanning

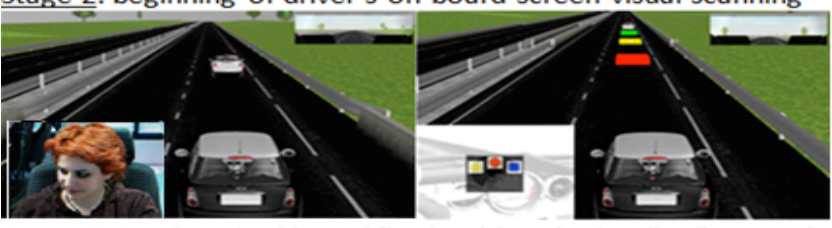

Stage 3: Lead car braking while the driver is visually distracted

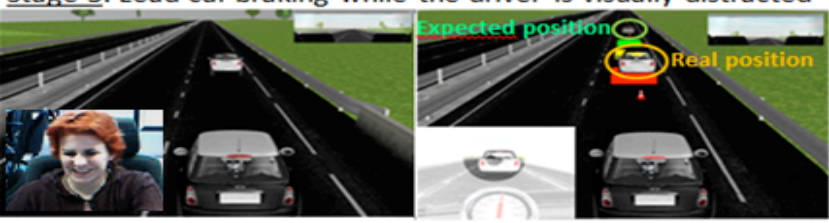

Stage 4: driver's eyes go back on road and she becomes aware of erroneous updating of her mental model while distracted

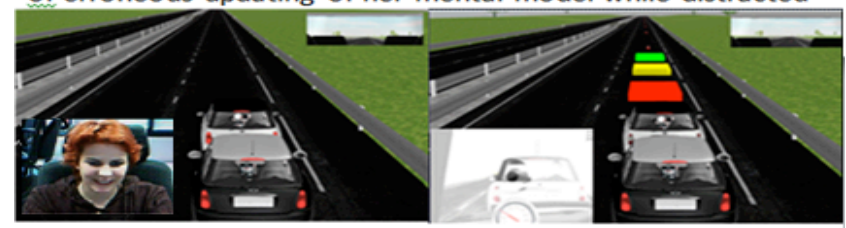

Stage 5: Crash (due to a too late reaction time)

Figure 9: Visual distraction effect simulations on driver's $S A$
This is typically what occurred in the example of crash initially presented in fig. 3, and then analyzed in Figure 8 and 9 from through COSMODRIVE simulations. These 2 Figures correspond to a simulation case for a similar driving scenario presented in fig. 3 (free following task). Like $58 \%$ of the observed human drivers, COSMODRIVE implemented here the first strategy for scanning of the additional screen (cf. fig. 2), requiring at last a long glance of 2 seconds. During these 2 seconds, COSMODRIVE manages the IVT with the lead car by using its mental model of the driving situation (see stages 2 on fig. 9). Unfortunately, the lead car brakes when the virtual eye is off-road and COSMODRIVE Situation Awareness becomes progressively totally different of the situational reality (stage 3 of Fig. 9). When the driver/model repays attention to the road scene (view $c$ on Fig.8 and stage 4 on Fig. 9), they suddenly become aware of the critical gap between the expected lead car position (as mentally assessed during the off-road glance by assuming a steady speed of the lead car) and the critical nature of the objective reality (as illustrated at stage 4 on fig. 9). Therefore, like the human drivers presented in fig. 2, the model immediately carried out an emergency braking (reaction time of $0.8 \mathrm{sec}$. on Fig 8), but the crash cannot be avoided.

\subsection{Conclusion and perspectives}

As illustrated in Fig. 9, such types of COSMODRIVE simulations allow us to in-depth investigate and understand what happens in the driver's mind when visually distracted: incomplete or incorrect perception of roadway cues due to off-road glances (required by the secondary task) can directly impact the formulation of an adequate Situation Awareness that will affect, in a second times, the driving performance. Through these cognitive simulation abilities of COSMODRIVE, it is expected in the future to explore visual distraction effects for a large set of driving scenarios, more particularly in terms of inadequate mental models, that is of a crucial interest for analysing human errors at both behavioural and cognitive levels, and for explaining some un-voluntary risk taking when drivers are distracted.

\section{Acknowledgments}

The research was supported by the European Commission 7th Framework Program, in the frame of ISi-PADAS Project.

\section{References}

Anderson, J. R., Bothell, D., Byrne, M. D., Douglass, S., Lebiere, C., \& Qin, Y . (2004). An integrated theory of the mind. Psychological Review, 111, (4), 1036-1060.

Baddeley A.D. (1986). Working Memory. Clarendon Press.

Bellet, T. (2011). Analysis, modeling and simulation of human operator's mental activities. In G. A. Boy (Ed), Handbook of Human-Machine Interaction, Ashgate.

Bellet, T., Bailly-Asuni, B., Mayenobe, P., \& Banet, A. (2009). A theoretical and methodological framework for studying and modelling drivers' mental representations. Safety Science, $47,1205-1221$. 
Bellet, T., Bailly, B., Mayenobe, P., \& Georgeon, O. (2007). Cognitive modelling and computational simulation of drivers mental activities. In P. Cacciabue (Ed.), Modelling Driver Behaviour in Automotive Environment: Critical Issues in Driver Interactions with Intelligent Transport Systems, 315-343, Springer Verlag.

Bellet, T., Mayenobe, P., Bornard, J.C., Gruyer, D. \& Mathern, B. (2010). COSMO-SIVIC: a first step towards a virtual platform for Human Centred Design of driving assistances. Proceedings of the $11^{\text {th }}$ IFAC/IFIP/IFORS/IEA Symposium on Analysis, Design, and Evaluation of Human-Machine Systems, Valenciennes, France

Bellet, T., Mayenobe, P., \& Bornard, J.C. (2010). How to investigate the living cognition: an application to dynamic simulation of mental activities while driving. In D. D. Salvucci \& G. Gunzelmann (Eds.), Proceedings of the 10th International Conference on Cognitive Modeling (pp. 1-6). Philadelphia, PA: Drexel University.

Donmez, B., Boyle, L. N., \& Lee, J. D. (2007). Safety implications of providing real-time feedback to distracted drivers. Accident; analysis and prevention, 39(3), 581-90.

Endsley, M. R. (1995). Toward a theory of situation awareness in dynamic systems. Human Factors 37, 32-64.

Gruyer D, Royère C, du Lac N, Michel G, and Blosseville JM, 2006. Sivic and rt-maps interconnected platforms for the conception and the evaluation of driving assistance systems. In: Proceedings of the ITS World Congress. London, UK.

Hall, E.T. (1966). The hidden dimension. New York: Doubleday.

Kontaratos, N.A. (1974). A system analysis of the problem of road casualties in the United States. Accident Analysis and Prevention, 6, 223-241.

Lee, J. D., Regan, M. A., \& Young, K. L. (2009). What drives distraction? Distraction as a breakdown of multilevel control. In M. A. Regan, J. D. Lee, \& K. L. Young (Eds.), Driver distraction: Theory, effects, and mitigation (pp. 42-56). Boca Raton, FL: CRC Press, Taylor \& Francis Group.

Mayenobe, P. (2004). Perception de l'environnement pour une gestion contextualisée de la coopération Homme-Machine. $\mathrm{PhD}$ Thesis, University of Clermont-Ferrand.

Michon, J.A. (1985). A critical view of driver behavior models : what do we know, what should we do ?. In: Evans, L., Schwing, R.C. (Eds), Human behavior and traffic safety. New York: Plenum Press.

Minsky, M. (1975). A Framework for Representing Knowledge. In P.H. Winston (Ed.), The Psychology of Computer Vision. New York: Mc Graw-Hill.

Neisser U (1976). Cognition and reality: principles and implications of cognitive psychology. WH Freeman.

Ochanine V.A. (1977). Concept of operative image in engineering and general psychology. In B.F. Lomov, V.F., Rubakhin \& V.F. Venda (Eds), Engineering Psychology. Moscow: Science Publisher.

Rasmussen J. (1986). Information processing and human-machine interaction: an approach to cognitive engineering. Amsterdam: North Holland.

Schilder, P. (1950). The image and appearance of the human body. New York: International Universities Press.

Schneider W., \& Shiffrin R.M. (1977). Controlled and automatic human information processing I: Detection, search and attention. Psychological Review, 84, 1-88.

Treat, J. R. (1980). A study of pre-crash factors involved in traffic accidents. HSRI Research Review, 10/11, 1-35
Victor, T., Harbluk, J. L., \& Engström, J. (2005). Sensitivity of eye-movement measures to in-vehicle task difficulty. Transportation Research Part F: Traffic Psychology and Behaviour, 8(2), 167-190.

Wierwille, W.W. Huise M.C., Fischer T.J., Dingus T.A. (1988) Strategic use of visual resources by the driver while navigating with an in-car navigation display system, FISITA XXIInd Congress. Automotive Systems Technology: the Future. Volume II. Technical papers Proceedings. Warrendale, PA: Society of Automotive Engineerrs, 2.661 - 2,675.

Wierwille, W. W. (1993). Visual and manual demands of in-car controls and displays. In B. Peacock \& W. Karwowski (Eds.), Automotive Ergonomics (pp. 299-320). London: Taylor \& Francis.

Zhang Y. (2011) Visual and Cognitive Distraction Effects on Driver Behavior and an Approach to Distraction State Classification. PhD Dissertation, North Carolina State University, Raleigh, USA.

Zintchenko P. (1966). Quelques problèmes de psychologie de la mémoire. In A. Leontiev, A. Luria \& A Smirnov (Eds), Recherches Psychologiques en URSS. Moscow: Editions du Progrès. 